LBL-36749

UC-414

\title{
Multidimensional Simulation Studies of the SELENE FEL Oscillator/Buncher Followed by a Radiator/Amplifier Output Scheme
}

\author{
S.J. Hahn* and W.M. Fawley** \\ *Stanford Linear Accelerator Center \\ Stanford University \\ Stanford, California 94309 \\ **Accelertor and Fusion Research Division \\ Lawrence Berkeley Laboratory \\ University of California \\ Berkeley, California 94720
}

February 1995

This work was supported by the Director, Office of Energy Research, Office of High Energy and Nuclear Physics, of the U.S. Department of Energy under Contract Nos. DE-AC03-76SF00015 (SLAC) and DE-AC03-76SF00098 (LBL). 


\section{DISCLAIMER}

This report was prepared as an account of work sponsored by an agency of the United States Government. Neither the United States Government nor any agency thereof, nor any of their employees, make any warranty, express or implied, or assumes any legal liability or responsibility for the accuracy, completeness, or usefulness of any information, apparatus, product, or process disclosed, or represents that its use would not infringe privately owned rights. Reference herein to any specific commercial product, process, or service by trade name, trademark, manufacturer, or otherwise does not necessarily constitute or imply its endorsement, recommendation, or favoring by the United States Government or any agency thereof. The views and opinions of authors expressed herein do not necessarily state or reflect those of the United States Government or any agency thereof. 


\section{DISCLAIMER}

Portions of this document may be illegible in electronic image products. Images are produced from the best available original document. 


\title{
Multidimensional simulation studies of the SELENE FEL oscillator/buncher followed by a radiator/amplifier output scheme*
}

\author{
S. J. Hahn ${ }^{\dagger}$ \\ Stanford Linear Accelerator Center, Stanford University \\ Stanford, CA 94309 \\ W. M. Fawley \\ Lawrence Berkeley Laboratory, University of California, \\ Berkeley, CA 94720
}

\begin{abstract}
We analyze and present numerical simulations of the so-called electron output scheme [G. I. Erg et al., 15th Int. FEL Conf., The Hague, The Netherlands, 1993, Book of Abstracts p. 50; Preprint Budker INP 93-75] applied to the SELENE proposal of using a high power FEL to illuminate satellite solar cells. In this scheme, a first stage FEL oscillator bunches the electron beam while a second stage "radiator" extracts high power radiation. Our analysis suggests only in the case where the radiator employs a long, tapered undulator will the electron output scheme produce a significant increase in extraction efficiency over what is obtainable from a simple, singlestage oscillator. 1- and 2-D numerical simulations of a 1.7 $\mu \mathrm{m} \mathrm{FEL} \mathrm{employing} \mathrm{the} \mathrm{electron} \mathrm{output} \mathrm{scheme} \mathrm{show}$ reasonably large bunching fractions $(\sim 0.3-0.4)$ at the output of the oscillator stage but only $\leq 2 \%$ extraction efficiency from the radiator stage.
\end{abstract}

Keywords: free-electron lasers, oscillators, amplifiers, tapered wigglers, multi-stage devices, power beaming

\section{INTRODUCTION}

Bennet et al. ${ }^{1}$ have recently proposed that a high power ( $\geq 1 \mathrm{MW}$ average power), ground-based Free Electron Laser (FEL) might be used to illuminate solar cells of orbiting satellites, providing a much larger electrical power capability than would be true from normal solar illumination only. In particular, the "electron output" scheme has been suggested as being particularly attractive for this application. In this scheme, a relatively low power oscillator in an optical klystron configuration is used to bunch the electron beam which is then transported

\footnotetext{
"This version of the paper has been revised slightly from that appearing in the proceedings of SPIE Photonics West '95 Conference.

tPermanent address: Dept. of Physics, Chung-Ang University, Seoul 156-756, Korea
} 
isochronously to a single pass undulator (the "radiator") where it produces large amounts of coherent radiation. Ideally, the oscillator portion of the device would produce quite large bunching fractions $(b \geq 0.5)$ at a sufficiently low power that neither mirror damage nor sideband generation would pose difficulties. We note that this scheme is somewhat similar to the double wiggler SAMOPA configuration proposed by LANL $^{2}$ in which output radiation from the oscillator is used as a high power seed to bunch the same electron beam in a single pass amplifier.

Last year, following up on previous modeling studies, ${ }^{3,4}$ we $\mathrm{e}^{5}$ examined with 1- and 2-D, time-dependent codes the optimization of cavity detuning and optical klystron dispersion lengths for stable performance of a possible experiment with the Novosibirsk microtron $\mathrm{FEL}^{6}$ at a wavelength of $8 \mu \mathrm{m}$. In the case of low single pass gain, we found that stable, single mode equilibria with moderately large bunching fractions $(b \approx 0.4)$ existed and that oscillator cavity power levels scaled inversely with dispersion lengths. In the case of high single pass gain $(G \geq 20)$, it became necessary to adopt large cavity losses to ensure single mode operation at reasonable power levels.

In this paper, we continue our study of the electron output scheme via analysis and numerical simulation. In $\xi 2$, we analytically compare the relative extraction efficiencies of the electron output scheme versus that obtainable from a "normal" oscillator FEL with outcoupling. Our results suggests that if mirror heat loading is not an issue, the overall complexity of the electron output scheme appears to outweigh its possible advantages in output power in much of parameter space. In §3, we outline the basic accelerator and FEL parameters using present-day technology for a hypothetical SELENE FEL operating at $1.7 \mu \mathrm{m}$, which corresponds to one of the more transparent, atmospheric "windows" of the near-infrared wavelength region (harmonic up-conversion to $0.85 \mu \mathrm{m}$ would be necessary for solar cell operation). In $\$ 4$, we present and discuss the results from numerical simulation modeling the performance of the system designed in $\S 3$. Our goal was high peak output laser power from the radiator while simultaneously keeping the power level on the oscillator mirrors acceptably low. We find that while relatively large output bunching fractions and low mirror power loading are possible in the oscillator stage, it may be difficult to extract more than a couple percent of the electron beam power into radiation unless a long, carefully-tapered wiggler is used in the radiator.

\section{THEORETICAL EXTRACTION EFFICIENCY OF THE ELECTRON OUTPUT SCHEME}

In order to compare the expected performance of the electron output scheme with that possible in more conventional FEL oscillators, we first introduce Colson' ${ }^{7}$ normalized quantities and discuss their values in a simple oscillator. We then analyze the properties of the radiator in the electron output scheme.

\subsection{Normalized Parameters}

Recognizing that the standard FEL equations could be scaled in the limit of $\gamma \gg 1$ and $\Delta \gamma \ll \gamma$, Colson introduced three quantities, $\nu, j$, and $a$ to represent the normalized particle energy (relative to resonant energy), current density, and transverse electric field, respectively.

$\nu$, defined by

$$
\nu \equiv 4 \pi N_{w}\left(\gamma-\gamma_{r}\right) / \gamma_{r}
$$

gives a particle's instantaneous rate of change in phase measured relative to that of a ponderomotive well corresponding to a plane wave:

$$
L_{w} \frac{\partial \theta}{\partial z}=\nu
$$

Here $N_{w}$ in the number of periods in an undulator of length $L_{w}$ where we have presumed a constant wavelength 
$\lambda_{w}$ and $\gamma_{r}$ is the resonant Lorentz factor. The normalized, complex field strength, $a$, defined by

$$
a \equiv \frac{4 \pi N_{w} e a_{w}[J J] L_{w}}{\gamma_{r}^{2} m c^{2}} \bar{E} \exp (i \phi)
$$

gives the instantaneous rate of change of $\nu$

$$
L_{w} \frac{\partial \nu}{\partial z}=-a \sin \psi
$$

Here $\vec{E}$ is the rms complex field strength, $a_{w}$ is the normalized rms vector potential, $[J J]$ is the Bessel function difference coupling term for a linearly polarized undulator, and $\psi \equiv \theta+\phi$. The total height (measured in units of in $\nu$ ) of the ponderomotive well is approximately $\pm 2|a|^{1 / 2}$ while the synchrotron wavenumber $\Omega_{y_{n}}$ is $L_{w} \Omega_{\text {syn }}=|a|^{1 / 2}$. Lastly, the normalized current density

$$
j \equiv \frac{8 n_{e} f N_{w}\left(e \pi a_{w}[J J] L_{w}\right)^{2}}{\gamma_{r}^{3} m c^{2}}
$$

gives the rate of change of the (complex) field

$$
L_{w} \frac{\partial a}{\partial z}=j(<\sin \psi>+i<\cos \psi>)
$$

where the brackets represent averaging over the particle phases. Here $f$ represents the effective filling fraction of the electron beam in the optical mode. In general, the electron beam radius $r_{b}$ is much less than the mode radius $\omega_{o}$ and $f \approx\left(r_{b} / \omega_{o}\right)^{2}$. With $k_{s} \equiv 2 \pi / \lambda_{s}$, when the Rayleigh range $Z_{R} \equiv k_{s} \omega_{o}^{2} / 2 \approx L_{w}$ as is generally true for low-to-moderate gain systems, one sees that $j \propto L_{w}^{2}$ rather than $L_{w}^{3}$ as is true in the 1D limit. In extremely high gain systems $(j \geq 100)$, one can operate with $Z_{R}$ much less than $L_{w}$ and the physics lies much closer to the $1 D$ limit of $f=1$. Practically speaking, however, most oscillators do not operate in this limit and the rest of our discussion presumes low-to-moderate single pass gain.

Field strengths in oscillators with untapered wigglers and moderate values of $j$ (i.e. $\leq 20$ ) saturate when $a \sim 10-20$ leading to an energy extraction efficiency (presuming single mode operation with negligible sidebands) of $\delta \gamma / \gamma \approx 1 / 2 N_{w}$. Although $a_{s a t}$ slowly grows with increasing $j$ (asymptotically as $j^{1 / 3}$ in the 1D limit), increasing $j$ by increasing $L_{w}$ decreases the net extraction efficiency until very large values of $j$ are reached. Moreover, if single mode operation is desired, the large sideband growth rate for large $j$ must be controlled which normally causes a lowering of extraction efficiency. Therefore, for purposes of comparison with the electron output scheme, let us define $L_{w}^{o}$ as the shortest undulator length that will permit adequate lasing for a given beam current, micropulse duration, emittance, and energy spread. Similarly, let $j^{\circ}$ be that given by expression (5) evaluated at $L_{w}=L_{w}^{o}$ and $f=f^{\circ} \equiv\left(k_{s} r_{b}^{2}\right) / 2 L_{w}^{o}$. Unless the cavity losses or beam energy spreads are large, $j_{0} \sim 1$. The corresponding "baseline" extraction efficiency of such an oscillator is then $\sim 1 / 2 N_{w}^{o}$.

\subsection{Energy Extraction Efficiency of a Radiator}

For purposes of predicting the overall extraction efficiency of the electron output scheme, let us ignore details of the oscillator and isochronous transport line to the radiator and presume that they are "well-designed", producing a bunching fraction $b \equiv \mid\left\langle e^{i \psi}>\right|$ lying in the (optimistic) range of $0.5-0.7$. We further presume that the radiator undulator is appropriately tapered to maximize $\langle\sin \psi\rangle$ with minimal particle detrapping from the ponderomotive well.

From examining the behavior of the on-axis optical field versus $z$ in a series of numerical simulations, the radiator has three distinct regions. The first region is relatively short with length $z_{1} \approx k_{s} r_{b}^{2} / 2$, the Rayleigh range corresponding to the electron beam radius. In this region, diffractive and refractive effects are small, the bunched portion of the beam can operate with $\psi$ in the range $\pi / 4$ to $\pi / 2$, and $a$ will grow linearly with $z$. 
Beyond $z_{1}$, diffraction must be controlled by exploiting "optical guiding" which arises from a positive $\langle\cos \psi\rangle$. Following the analysis presented in Scharlemann, Sessler, and Wurtele ${ }^{8}$ we expect strong, refractive guiding when the "fiber parameter"

$$
V^{2} \equiv\left(n^{2}-1\right) k_{s}^{2} r_{b}^{2}
$$

is of order 1 or greater. The real part of the index of refraction $n$ is given by

$$
\operatorname{Re}(n)-1=\frac{j}{k_{z} L_{w}|a|}<\cos \psi>
$$

where $a$ refers to the on-axis normalized field strength and $j$ is evaluated with $f=1$ (this eliminates the actual dependence of $n$ upon $L_{w}$ since then $j \propto L_{w}^{3}$ and $\left.a \propto L_{w}^{2}\right)$. In the usual FEL regime where $(n-1)$ is small, one finds

$$
V^{2}=4 \frac{j}{|a|} \frac{k_{3} r_{b}^{2}}{2 L_{w}}<\cos \psi>
$$

At this point, let $L_{w} \rightarrow L_{w}^{0}$ (even though the actual radiator length might be many times this) and use the definition of $j^{\circ}$ from the end of the previous section. This results in

$$
V^{2}=4 \frac{j^{\circ}}{|a|}\langle\cos \psi>
$$

For sufficiently small $|a|$, the optical guiding is strong and, as was true in the first region, $|a|$ continues to grow linearly with the product $z\langle\sin \psi\rangle$.

Eventually though, when $|a|$ approaches

$$
a^{*} \equiv 4 \Gamma j^{0}<\cos \psi>
$$

$V^{2}$ becomes sufficiently smaller than 1 that optical guiding "fails", and the radiation begins to leak significantly beyond $r=r_{b}$. Here $\Gamma \sim 1-1.5$ takes into account $n$ is actually complex, At this point, we enter the third region where $|a| \approx a^{*}$ remains nearly constant on axis and, since the particle deceleration is directly proportional to $a \sin \psi[\mathrm{Eq}$. (4)], the total power grows linearly with $z$, as opposed to guadratically as was true in the first two regions. (Interestingly, our colleague K.-J. Kim has pointed out that in the case of purcly spontaneous emission from a pre-bunched beam, the power also grows linearly with $z$ and appears to be equivalent to $\langle\cos \psi\rangle=0$ and a positive $\left\langle\sin \psi>\right.$ ). The total energy extraction in the third section of the radiator with length $z_{3}$ is thus determined as

$$
\frac{\Delta \gamma}{\gamma}=\frac{\Delta \nu}{4 \pi N_{w}^{\circ}} \approx\left(\frac{z_{3}}{L_{w}^{\circ}}\right) \frac{1}{2 N_{w}^{\circ}}\left(\frac{2 \Gamma j^{\circ}}{\pi}\right)\langle\sin \psi\rangle\langle\cos \psi\rangle
$$

where we have presumed, optimistically, that the bunching fraction has remained constant. Since the maximum value of $\langle\sin \psi\rangle\langle\cos \psi\rangle$ is $b^{2} / 2$, it appears that the radiator wiggler is less than half as efficient per unit length as would be a "normal" oscillator with $j^{\circ} \approx 1$ in terms of energy extraction. When energy spread, detrapping, and time-dependent effects (in particular, slippage) are taken into account, the relative efficiency may be much smaller. Consequently, only if $L_{w}^{o}$ is quite small such that radiator lengths approaching $10 L_{w}^{0}$ or greater are physically reasonable does the electron-output scheme appear to lead to a significant increase in overall extraction efficiency when compared with what is far more easily produced by a "normal" FEL oscillator if mirror survivability is not an issue.

\section{A HYPOTHETICAL $1.7 \mu \mathrm{m}$ SELENE SYSTEM}

In this section we outline an hypothetical, SELENE FEL subsytem operating at $\lambda_{s}=1.7 \mu \mathrm{m}$ using available technology. A full system design must, of course, include details such as beam transport, energy recovery, RF stability, etc.. Our interest here was to see what sort of extraction efficiency might be possible with "believable" parameters. 


\subsection{Accelerator and Electron Beam Parameters}

The accelerator is a photocathode-equipped RF linac operating at a frequency of $2856 \mathrm{MHz}$, similar to that operating at Brookhaven. ${ }^{10}$ Micropulse parameters at entrance to the FEL undulator are 100-MeV energy, 0.25-1.0 nC charge contained in a parabolic micropulse of 2.5-ps FWHM duration (i.e. $I_{\text {peak }} \sim 100-400 \mathrm{~A}$ ). The equivalent, micropulse-averaged beam powers are 71 and $285 \mathrm{MW}$ respectively. We adopted a normalized longitudinal emittance of $0.25 \mathrm{ps}-\mathrm{MeV}$ (i.e. $\delta \gamma / \gamma=0.1 \%$ ), and a normalized transverse emittance of $\leq 10 \pi \mathrm{mm}$ mrad. Both the transverse and longitudinal phase spaces were assumed to have Gaussian distributions.

\subsection{Oscillator Undulator and Cavity Parameters}

We have chosen a linearly-polarized undulator with a constant wavelength $\lambda_{w}=4 \mathrm{~cm}$. The corresponding $a_{w}$ and peak on-axis magnetic field strengths in the oscillator are 1.5 and $0.57 \mathrm{~T}$ respectively. We have adopted equal strength focusing in the wiggle plane (as might be provided by curved pole faces or quadrupoles) which results in an equilibrium e-beam radius of $0.35 \mathrm{~mm}$. At the midpoint of the undulator is a dispersive section with an effective length ${ }^{7}$ of $D L_{w}$ whose major purpose is to reduce the steady-state power level and mirror loading. The instantaneous energy spread of $\Delta \gamma=0.22$ limits $(1+D) L_{w}$ to $\approx 4.0 \mathrm{~m}$. Higher values led to a significant reduction in output bunching of the electron beam and thus lower power in the radiator section. We note that in a "real" system, micropulses contain both an instantaneous and correlated energy spread and that the limits on $(1+D) L_{w}$ will be affected by both. We picked a cavity length of $10 \mathrm{~m}$, corresponding to a $15-\mathrm{MHz}$ round-trip frequency. For the following numerical simulation studies, we presumed a constant pass-to-pass cavity power loss rate of $10-70 \%$ that was independent of the transverse field profile.

\subsection{Radiator Parameters}

We presumed that beam transport from the end of the oscillator undulator to the beginning of the radiator was "perfect" and preserved exact details of the transverse and longitudinal phase space. As with the oscillator, for the radiator we chose a constant, $\lambda_{w}=4 \mathrm{~cm}$ period, linearly-polarized undulator but tapered the magnetic field strength to increase the extraction efficiency. For the following simulations, we computed the taper in a time-independent run with $I=I_{\text {peak }}$ using the "self-design" feature of the $2 \mathrm{D}$ GINGER code. The taper began approximately $1.5 \mathrm{~m}$ into the radiator wiggler which allows the initially zero-strength electromagnetic field to build up and decelerate the bunched particles approximately one ponderomotive well height.

\section{SIMULATION RESULTS}

As in our previous paper, ${ }^{5}$ we used the FEL1D and GINGER time-dependent simulation codes to model the hypothetical SELENE system of above. The 1D simulations were done without energy spread and presumed a constant filling fraction $f=r_{b}^{2} / \omega_{0}^{2}$. The 2D simulations included energy spread but due to CPU time considerations only modeled the first couple hundred passes of the oscillator (sufficient to obtain saturation when starting from shot noise) before taking the bunched electron beam of the last pass into the radiator undulator.

Our first series of simulations used $I_{\text {peak }}=100 \mathrm{~A}$ and $L_{w}=2 \mathrm{~m}$ resulting in $j_{0}=5.6$ and a single-pass gain of about 6 for a dispersion section strength $D L_{w}=2 \mathrm{~m}$. In Fig. la we plot the micropulse-averaged output power for the oscillator for different cavity loss rates versus various values of the cavity detuning length $\delta L$. When $-\delta L$ is too small, the output signal becomes temporally chaotic due to sideband growth. As is evident from the figure, smaller and smaller values of $-\delta L$ are necessary for stabilization as the cavity loss rate increases. Fig. $1 \mathrm{~b}$ 

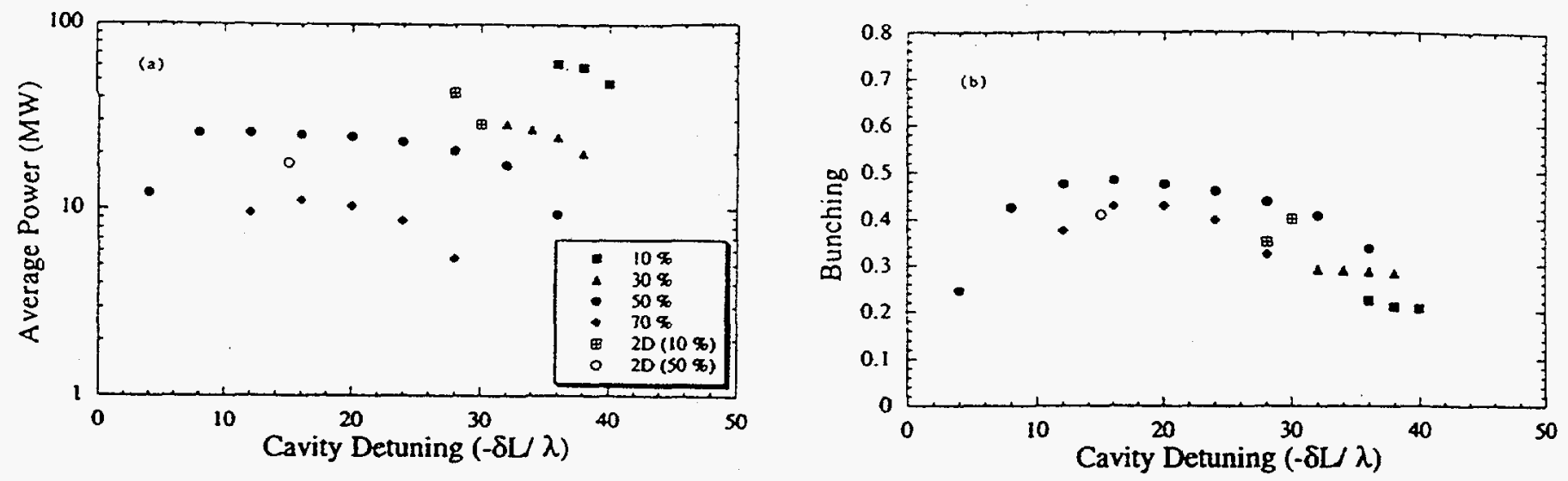

Figure 1: Micropulse-averaged output power (a) and electron beam bunching fraction (b) versus cavity detuning length as computed by the FELID code for the oscillator stage of a $1.7 \mu \mathrm{m}$ SELENE FEL with $I_{\text {peak }}=100 \mathrm{~A}$ and a 2.5-ps FWHM micropulse duration. The different curves refer to single pass power losses in the cavity. The points marked "2D" refer to results from the GINGER code which include energy spread, emittance, and diffraction effects.

plots the micropulse-averaged value of the output bunching fraction. The maximum value of $b \approx 0.45$ occurs for relatively large cavity loss values. The $2 D$ GINGER simulations generally confirm the $1 D$ results but are shifted to somewhat smaller values of $-\delta L$ and $\langle P\rangle$.

For the GINGER simulation of the "integrated" oscillator and radiator at $100-A$ current, we choose $-\delta L=28 \lambda$ with a cavity loss rate of $10 \%$. This optimized stability without too seriously degrading bunching in the tail half of the electron beam pulse. Figure 2 a plots the micropulse-averaged field power and bunching strength versus $z$ in the radiator. From Eq. (12) with $b^{2} \approx 0.1$, the predicted energy extraction over the last $6 \mathrm{~m}$ of the radiator is about $1.5 \%$, or $150 \mathrm{MW}$ as compared with the simulation result of $65 \mathrm{MW}$. However, most of the simulation output power is contained in a peak of $150 \mathrm{MW}$ of about 0.8 ps duration so slippage (which totals $1.1 \mathrm{ps}$ ) is probably playing an important role. While a more optimized oscillator and radiator design might lead to an increase of $b^{2}$ to perhaps as much as 0.2 , it would appear to be very difficult to extract more than $\approx 5 \%$ of the beam energy unless one considers radiator lengths in excess of $10 \mathrm{~m}$. Slippage effects in such a long wiggler will be even worse, however. By contrast, a simple oscillator with $50 \%$ hole outcoupling and $L_{w}=1.2 \mathrm{~m}\left(N_{w}=30\right)$ should give $1.5 \%$ extraction efficiency, if the mirrors can survive the heat loading. The output spectrum (Fig. 2b) is quite narrow and nearly all the power is contained in a spectral width of $\pm 1 \%$ or less. Sidebands, if any, are of negligible strength. Consequently, we would not foresee any difficulties in beaming this power through the atmosphere.

Similar simulations were done for the case of $I_{\text {peak }}=400 \mathrm{~A}$. With the higher current, we reduced the oscillator wiggler length to $0.96 \mathrm{~m}$ and, in order to reduce saturated power levels, included a dispersion section of strength $D L_{w}=1.92 \mathrm{~m}$. A $50 \%$ cavity loss rate and $-\delta L=10.8 \lambda$ reduced the effective single pass gain to 0.7 and led to a saturated cavity power of $110 \mathrm{MW}$ and an average output bunching fraction of 0.33 . With neither such a high cavity loss nor a dispersive section, the saturated cavity power wonld be more than an order of magnitude large. Thus, as before, mirror damage will be much less a worry than would be the case in a simple oscillator.

As compared with the lower current example, however, we found it difficult to obtain a stationary mode output for $I_{\text {peak }}=400 \mathrm{~A}$ with minimal sideband strength. The time-resolved bunching at the oscillator output shows 3-4 oscillations with a peak-to-peak amplitude of $0.15-0.2$ over then central 2 ps of the micropulse. These oscillations are associated with sidebands which contain about $1 \%$ of the output laser power from the oscillator. In a "real" system, one might use gratings to minimize the growth of these sidebands in the oscillator since their effects on the bunching provides a seed for sideband growth in the radiator. 

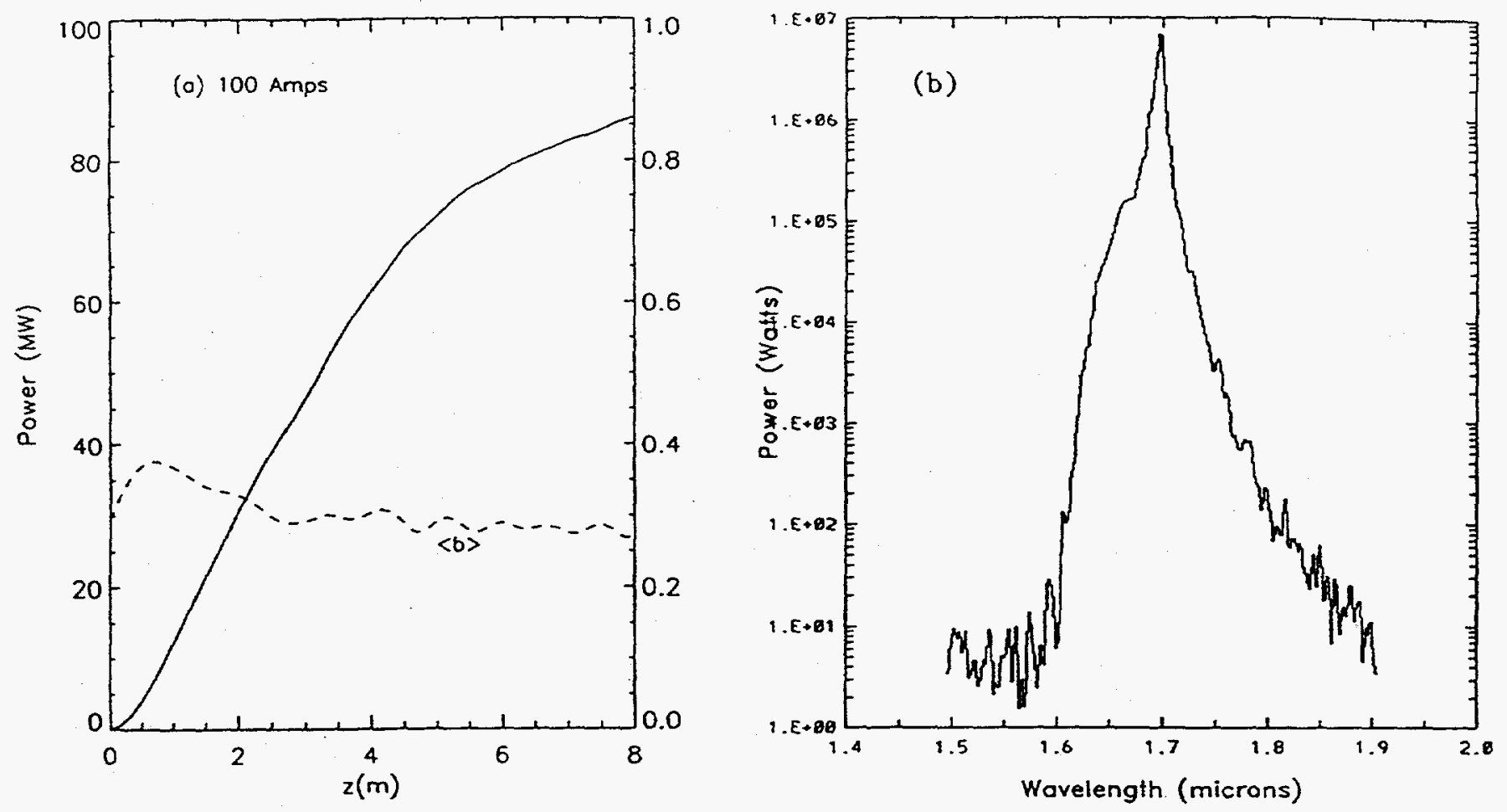

Figure 2: (a) Micropulse-averaged output power and electron beam bunching fraction versus $z$ in the radiator stage of a $1.7 \mu \mathrm{m}$ SELENE FEL with $I_{\text {peak }}=100 \mathrm{~A}$ and a 2.5 -ps micropulse duration as computed by the $2 \mathrm{D}$, time-dependent GINGER code. A 3\%, approximately linear taper of $a_{w}$ began $1.5 \mathrm{~m}$ into the 8-m long undulator. (b) Predicted output spectrum (plotted semi-logarithmically) at the end of the radiator.

The higher current permits beginning the taper at $z=1 \mathrm{~m}$ in the radiator undulator and also a larger net decrease of $a_{w}$ of about $7.5 \%$ over a $10-\mathrm{m}$ length. By the end of the radiator, the average power reached $720 \mathrm{MW}$ (or $1.8 \%$ extraction efficiency) while the bunching fraction decreased slightly from 0.30 to 0.24 as shown in Fig. 3a. Between $z=2 \mathrm{~m}$ and $z=6 \mathrm{~m}$ the power increased by $350 \mathrm{MW}$ or, equivalently, an extraction rate of of $0.22 \%$ per meter. For comparison, expression (12) predicts $0.4 \%$ per meter after adopting an average bunching fraction of 0.28 . As before, the output spectrum (Fig. $3 \mathrm{~b}$ ) is relatively narrow and but the sideband strength is now about $10 \%$ of fundamental. Inspection of the two-dimensional surface $b(z, t)$ shows that the bunching fluctutations present at the oscillator output evolve in the radiator into three well-bunched $(b \approx 0.45)$ temporal regions of duration $0.2 \mathrm{ps}$ in the central $1.5 \mathrm{ps}$ of the micropulse. Both the front and tail 0.5 ps regons of the micropulse appear to be completely detrapped after the $2-\mathrm{m}$ point in the radiator undulator. It is conceivable that, with proper optimization and sideband control in the oscillator, the extraction rate might be increased to somewhere between 0.4 and $0.8 \%$ per meter, in which case efficiencies above $5 \%$ in a $10-\mathrm{m}$ radiator would be possible. If so, the electron output scheme would then appear to be advantageous over simple oscillator configurations.

\section{CONCLUSIONS}

The electron output scheme is, in theory, an innovative way to overcome limitations due to oscillator mirror damage and extract large amounts of coherent FEL radiation. With a reasonable value of cavity loss and the addition of dispersion sections to the oscillator first stage, the intracavity power can be reduced by an order of magnitude or more compared with what might exist in a simple, one-stage oscillator. According to 1 - and 

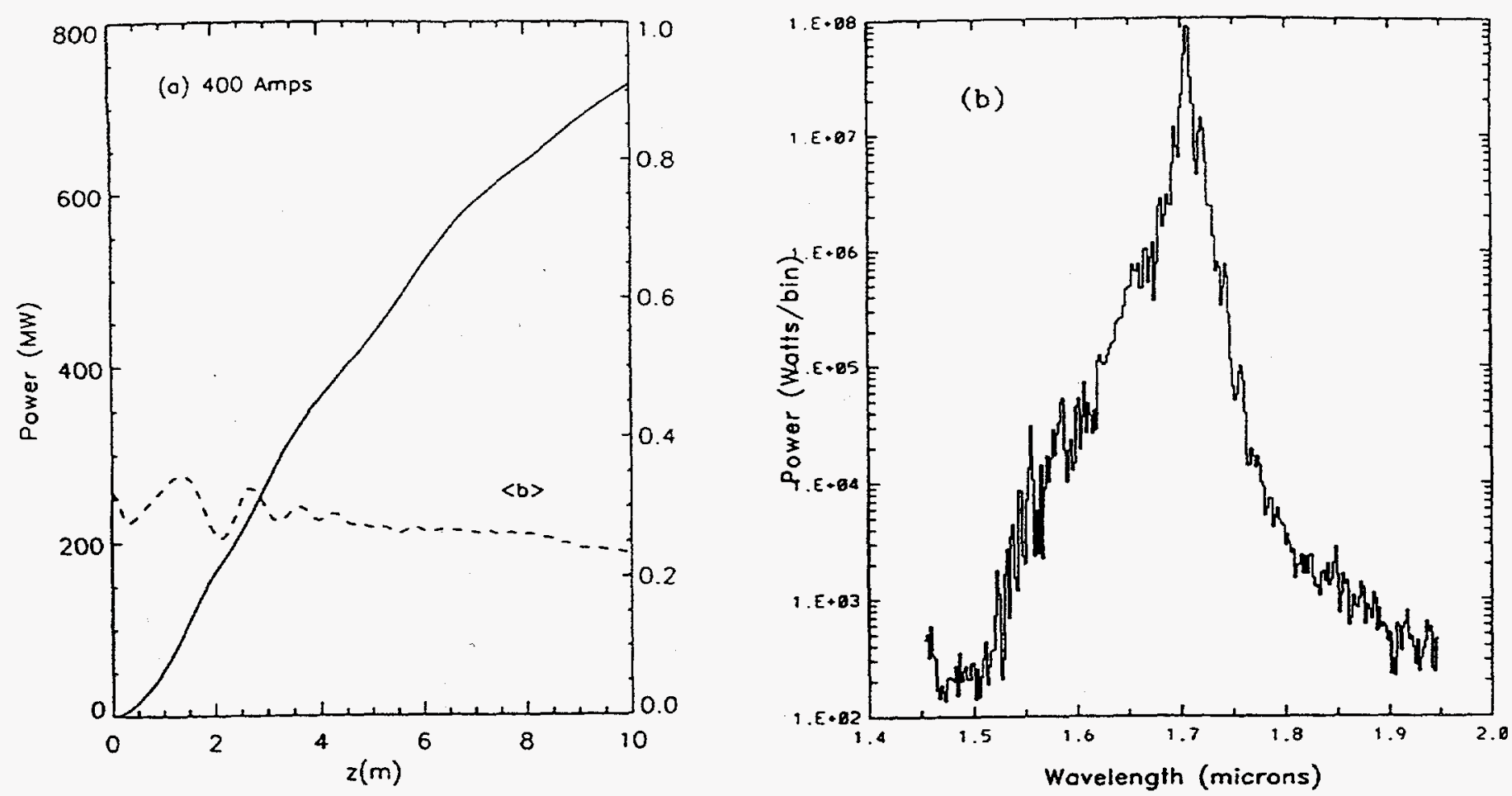

Figure 3: (a) GINGER predictions for the micropulse-averaged output power and electron beam bunching fraction versus $z$ in the radiator stage of a $1.7 \mu \mathrm{m}$ SELENE FEL with $I_{\text {peak }}=400 \mathrm{~A}$. A $7.5 \%$, approximately linear taper of $a_{w}$ began $1.0 \mathrm{~m}$ into the $10-\mathrm{m}$ long undulator. (b) Predicted output spectrum (plotted semi-logarithmically) at the end of the radiator.

2-D, time-dependent simulation results, it should be possible to construct a SELENE first stage with available technology that would operate in a stationary mode with an output bunching fraction $b \sim 0.3-0.45$. In one low current example, we have obtained approximately $1 \%$ extraction efficiency from the radiator stage with spectral widths of $1 \%$ or less with no evidence of sideband formation. A higher current example shows $2 \%$ extraction efficiency but at the cost of sideband formation; such sidebands might be lessened or eliminated via gratings in the oscillator stage. In the radiator stage, it will be difficult to extract more than a couple percent of the beam power unless the length is 5-10 times greater than what would be necessary for a simple oscillator. Due to the limitations of "optical guiding", our analysis suggests that the power grows linearly with $z$ over the majority of the radiator undulator. The radiator undulator field must be carefully tapered in order to minimize particle detrapping from the ponderomotive wells because the power extraction scales as $b^{2}$. The ultimate SELENE goal of a MW-class average power laser in the near-infrared, if based on an RF-accelerator driven FEL, is certainly not a trivial undertaking.

\section{ACKNOWLEDGEMENTS}

We are pleased to acknowledge useful discussions with our colleague K.-J. Kim. This work was supported by the Director, Office of High Energy and Nuclear Physics, U.S. Department of Energy, under Contracts No. DE-AC03-76SF0015 (SLAC) and DE-AC03-76SF00098 (LBL). 


\section{REFERENCES}

[1] H. E. Bennett et al., "Free-electron laser power beaming to satellites at China Lake, California", Nucl. Inst. and Meth., A341, pp. 124-131, 1994.

[2] J. C. Goldstein, B. E. Carlsten, and B. D. McVey, "INEX Simulations of the Los Alamos HIBAF Free-Electron Laser MOPA Experiment", Nucl. Inst. and Meth., A296, pp. 273-281, 1990, and references therein.

[3] D. D. Quick, J. Blau, R. K. Wong, and W. B. Colson, "Phase space simulation of electron bunching and power output in the Novosibirsk/SELENE FEL", Nucl. Inst. and Meth., A341, ABS 92, 1994.

[4] J. Blau, R. K. Wong, D. D. Quick, and W. B. Colson, "Three dimensional simulations of the Novosibisk/SELENE FEL", Nucl. Inst. and Meth., A341, ABS 94, 1994.

[5] S.J. Hahn, W.M. Fawley, K.-J. Kim, and J.A. Edighoffer, "Time-dependent multi-dimensional simulation studies of the electron output scheme for high power FELs", Proc. 16th Int. FEL Conf., in press, Nucl. Inst. and Meth., 1995.

[6] G. I. Erg et al., 15th Int. FEL Conf., The Hague, The Netherlands, (1993); Preprint Budker INP 93-75.

[7] W.B. Colson, "Classical free electron laser theory", in Laser Handbook (Free Electron-Lasers), ed. W.B. Colson, C. Pellegrini, and A. Renieri, 6, pp. 115-194, North-Holland, Amsterdam, 1990.

[8] E.T. Scharlemann, A.M. Sessler, and J.S. Wurtele, "Optical guiding in a free electron laser", Nucl. Inst. and Meth., A239, pp. 29-35, 1985.

[9] R.A. Jong, W.M. Fawley, and E.T. Scharlemann, "Modeling of induction-linac based free-electron laser amplifiers" in Modeling and simulation of laser systerns, SPIE 1045, pp. 18-27, 1989.

[10] K. Batchelor et al., "Performance of the Brookhaven photocathode rf gun", Nucl. Inst. and Meth., A318, pp. 372-376, 1992. 